\title{
Logistic population growth and beyond: the influence of advection and nonlocal effects
}

\author{
Emilio Hernández-García and Cristóbal López \\ Instituto Mediterráneo de Estudios Avanzados IMEDEA* (CSIC-UIB), Campus \\ de la Universitat de les Illes Balears, E-07122 Palma de Mallorca, Spain.
}

\section{Introduction}

By introducing the logistic equation in the context of demographic modelling [1], J.F. Verhulst made seminal contributions to at least two important fields of research: On the one hand, to the quantitative approach to Population Dynamics, a subject in which substantial development beyond Verhulst ideas only occurred 100 years after him. On the other, to the basics of Nonlinear Science through a detailed study of the predictions of a nonlinear model and its comparison with experimental data.

The dynamics of biological populations in aquatic environments $[2,3,4]$ is an excellent framework to see recent developments in which these disciplines work together. Growth, limitation, competition, predation and all the other kinds of biological interactions appear in this context, and nonlinear processes are relevant both in the biological dynamics and in the motion of the turbulent fluid in which the population lives.

In this contribution we present two examples in the above field. In both cases a prominent role is played by the logistic growth process (i.e. population growth limited by finite resources), but other ingredients are also included that strongly change the phenomenology. In Section 2, a phytoplankton population experiencing logistic growth is studied, but in interaction with zooplankton predators that maintain it in a state below the carrying capacity of the supporting medium. In the appropriate parameter regime the system behaves in an excitable way, with perturbations inducing large excitationdeexcitation cycles of the phytoplankton population. The excitation cycles become strongly affected by the presence of chaotic motion of the fluid containing the populations.

In section 3, an individual based model of interacting organisms is presented, for which logistic growth is again the main ingredient. Reproduction of a given individual is limited by the presence of others in a neighborhood of finite size. This nonlocal character of the interaction is enough to produce an instability of the basic state of particles homogenously distributed, and clustering of the individuals occurs, which form groups arranged in an hexagonal lattice (when the population lives in a two-dimensional space).

\footnotetext{
${ }^{\star}$ http://www.imedea.uib.es/PhysDept
} 


\section{Plankton dynamics driven by an environmental open flow}

Plankton is the generic name given to a large number of aquatic organisms, most of them microscopic, living in the oceans, lakes or rivers, and characterized by the fact that they are transported by water currents in an approximately passive manner [2]. A major distinction in this group should be made between phytoplankton, the organisms with photosynthetic capabilities, that can grow from nutrients in the water and light, and zooplankton, the small predators that consume them. This distinction leads to the simplest models of aquatic population dynamics, which take into account just these two trophic levels.

Here we consider the phytoplankton-zooplankton competition model proposed in [5]. Phytoplankton growths logistically, and in addition it is limited by the zooplankton grazing, which itself dies grazed by upper trophic levels not explicitly modelled. The fundamental feature of the model is its excitable behavior. In just a few words, this means that activator and inhibitor variables can be identified. The activator (phytoplankton in our case) displays some kind of autocatalytic growth behavior, but the presence of the inhibitor (zooplankton) controls it so that the dynamical system has a stable fixed point of low phytoplankton population as the unique global attractor. The essence of the excitability phenomenon is the presence of a threshold, such that if the system is perturbed above it, variables reach the stable fixed point only after a large excursion in phase space in which phytoplankton population grows to values close to the carrying capacity of the medium. This behavior usually appears when the activator has a temporal response much faster than the inhibitor, which then takes some time before stopping the growth of the activator. Observed plankton bloom phenomena have been interpreted in this dynamical context $[5,4]$.

Explicitly, the dynamics of the space- and time-dependent phytoplankton, $P=P(\mathbf{x}, t)$, and zooplankton, $Z=Z(\mathbf{x}, t)$, concentrations is ruled by

$$
\begin{aligned}
\frac{\partial}{\partial t} P+\mathbf{v} \cdot \nabla P-D \nabla^{2} P & =r\left[\beta P\left(1-\frac{P}{K}\right)-f(P) Z\right] \\
\frac{\partial}{\partial t} Z+\mathbf{v} \cdot \nabla Z-D \nabla^{2} Z & =r \epsilon[f(P) Z-\omega Z] .
\end{aligned}
$$

The left-hand-side terms represent the transport processes: both species are advected by the same fluid flow characterized by the velocity field $\mathbf{v}=\mathbf{v}(\mathbf{x}, t)$, that we assume to be incompressible and not altered by the back-influence of the biological concentration fields. We choose $\mathbf{v}$ to be two-dimensional to emphasize the role of horizontal transport [3], and defined on a twodimensional square box domain $\Omega=[0,2 L] \times[-L, L]$ with Cartesian coordinates $\mathbf{x}=(x, y)$. The diffusion operator $D \nabla^{2}$, assumed to be the same for both species, models small-scale complex turbulent motions not explicitly included in $\mathbf{v}$. The right-hand-side contains the biological interactions 
terms: $r$ controls the ratio of the transport time scales to the biological activity time scales, $\epsilon$ sets the ratio of phytoplankton- to the much larger zooplankton-growth time scale, $K$ is the phytoplankton carrying capacity, $\beta$ the phytoplankton growth rate, $\omega$ a linear zooplankton mortality, and

$$
f(P)=\frac{P^{2}}{P_{0}^{2}+P^{2}}
$$

is a Hollings type III response function, describing the zooplankton predation on phytoplankton. As demonstrated in [5], is the functional form in (2) the responsible for the excitable character.

Following [5] we take non-dimensional units such that $\beta=0.43, K=1$, $\epsilon=0.01, P_{0}=0.053$, and $\omega=0.34$. This corresponds to phytoplankton doubling times of the order of days, and zooplankton time scales in the range of months. Biological concentrations have been scaled so that the phytoplankton carrying capacity (of the order of $100 \mu \mathrm{g}$ of Nitrogen equivalent per liter) is the unity. For these parameter values the biological dynamical system is in the excitable regime. Excitable behavior generally appears for $\epsilon$ small enough, which biologically means that, as already stated, time scales for phytoplankton growth are much shorter than for zooplankton. This is the biologically relevant case. We study the influence of transport by varying its relative strength via the parameter $r$. $D$ is fixed to $10^{-5}$ and $L=9$, which means that the diffusive spatial scale corresponding to the phytoplankton doubling time is between three and four orders of magnitude smaller than system size.

Since the velocity field $\mathbf{v}=\left(v_{x}, v_{y}\right)$ is two-dimensional and incompressible it can be written in terms of a streamfunction $\Psi(x, y, t)$ :

$$
\begin{aligned}
& v_{x}=\frac{\partial \Psi}{\partial y} \\
& v_{y}=-\frac{\partial \Psi}{\partial x}
\end{aligned}
$$

We consider the following streamfunction $[6]$ :

$$
\Psi=\Psi_{0} \tanh \left(\frac{y}{d}\right)+\mu \exp \left(-\frac{(x-L)^{2}+y^{2}}{2 \sigma^{2}}\right) \cos (k(y-v t)) .
$$

It represents an oceanic jet perturbed by a localized wave-like feature, trapped by topography or some geographical accident. The first term is the main jet, of width $d$, flowing towards the positive $x$ direction with maximum velocity $\Psi_{0} / d$ at its center. The wave-like perturbation, of strength $\mu$, is represented by the second term. It is localized in a region of size $\sigma$ around the point $(x, y)=(L, 0)$, and the wavenumber and phase velocity (directed towards the positive $y$ direction) are $k$ and $v$, respectively. The complete velocity field is time-periodic with period $2 \pi / k v$. 
It is very important to note that the region $\Omega$ is open with respect to this flow, so that we have the situation of chaotic scattering [7]: particles enter $\Omega$ from the left, following essentially straight trajectories, experience the irregular motion called transient chaos [7] when reaching the wave region (which in consequence becomes a mixing region), and finally they leave the system. For $\mu$ large enough, recirculation in the mixing region gives birth to a chaotic saddle in $\Omega$. The chaotic saddle is the (fractal) subset of the mixing region where particles are trapped forever. It is formed by an infinite number of bounded hyperbolic orbits in the mixing region. The stable manifold of the saddle contains orbits coming from the inflow region but never escaping from the mixing zone. Concerning the unstable manifold, if a droplet of dye (or any other passive tracer) is injected into the mixing region, most of it will be advected downstream in a short time. But part of the dye will remain close to the chaotic saddle for very long times, and continuously ejected along its unstable manifold. In this way passive tracers such as dye traces out the unstable manifold of the chaotic saddle, giving rise to the fractal patterns characteristic to open flows. We next study how these flow structures affect the plankton dynamics given by the right-hand-side of Eq. (1). Pictures of the streamfunction and of the chaotic saddle can be seen in [6].

Equations (1) are solved by a semilagrangian method. The fixed point representing stable phytoplankton-zooplankton coexistence in the absence of flow and diffusion is given by $P=P_{e}$ and $Z=Z_{e}$, with $P_{e}=P_{0} \sqrt{\sigma /(1-\sigma)}=$ 0.03827 and $Z_{e}=\beta\left(1-P_{e} / K\right)\left(P_{0}^{2}+P_{e}^{2}\right) / P_{e}=0.04603$. We choose these values to be imposed as Dirichlet boundary conditions on the boundary of $\Omega$. In this way fluid particles enter in the system with a plankton content corresponding to the equilibrium concentrations, which is a rather natural condition from the biological point of view. During an excitation phase, the values of phytoplankton concentration rise to $P \approx 0.8-0.9$

Since $\left(P_{e}, Z_{e}\right)$ is a stable equilibrium point, dynamics will be trivial without an initial seed to trigger the excitation dynamics. Our initial condition is a localized patch of high phytoplankton concentration close to the left part of $\Omega: P(\mathbf{x}, t=0)=P_{e}+Q \exp \left[-\left(\left(x-x_{0}\right)^{2}+y^{2}\right) / l^{2}\right], Z(\mathbf{x}, t=0)=Z_{e}$. We take $Q=0.5, x_{0}=0.3 L$, and $l=0.11 L$. The jet transports the patch towards the scattering or mixing region, where interesting dynamics occurs. The flow parameters are $d=1, \Psi_{0}=2, \sigma=2, k=1$, and $v=1$, giving a flow period $T=2 \pi / k v=2 \pi$.

We now consider $\mu=3$ (a chaotic saddle is present in the system above $\mu \approx 2$ ). For small $r$, the biological dynamics is slow compared to the time scales of stirring by the flow. The phytoplankton patch is strongly deformed when reaching the scattering region. Plankton is stretched into long and thin filaments that become rapidly diluted into the surrounding unexcited fluid by the effect of diffusion. Thus excitation is destroyed by the fast stirring and by diffusion. Increasing $r$, i.e. by making the biological dynamics faster or the flow slower, a dramatic change occurs. The transition to the new 
regime occurs around $r \approx 1$. Plankton is again stretched in filaments but the width stabilizes and the excitation becomes distributed in the system, without leaving it (it remains forever) and oscillating in shape following the period of the flow. Some features of the distributions of both phytoplankton and zooplankton (see Fig. 1) seem to mimic the shape of the unstable manifold of the chaotic saddle, so that in these zones we can say that plankton is basically covering it with a finite width. Summing up, the relevant result we have shown is the following: transient chaos, characteristic of open flows, plus transient excitation, characteristic of excitable systems, give rise to a permanent pattern of high biological activity (excitation).

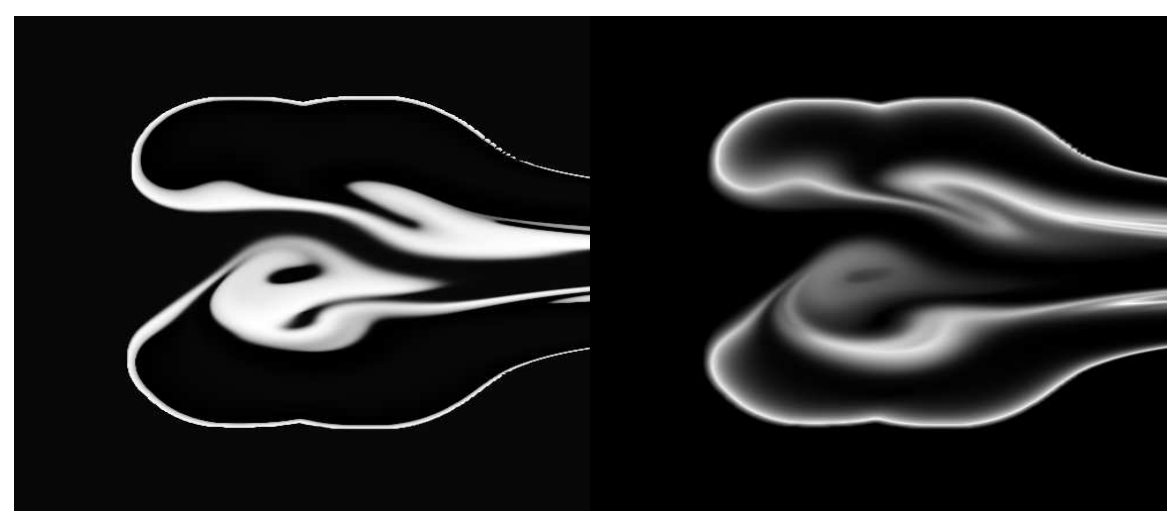

Fig. 1. Distribution of phytoplankton (left) and zooplankton (right) at time $t=100$ and parameters $r=10$ and $\mu=3$. Dark grey corresponds to low concentration and lighter grey to higher concentration. A state of permanent excitation is sustained in the region close to the chaotic saddle and its unstable manifold (being the domain far from this region in the unexcited equilibrium state). The shape of the distributions changes in time with the period of the flow.

The explanation for the observed behavior can be elaborated along the lines of previous works $[8,9]$ as follows: The tendency of the chaotic flow to stretch fluid elements into long and thin filaments competes with the effect of diffusion and biological growth, which tends to expand excited regions, so that a compensation can be achieved in some parameter range. Above a given biological growth rate, steady filament solutions appear via a saddlenode bifurcation. This can be explicitly shown in simplified models capturing some of the features of the full system (1). When this steady solution does not exist, initial perturbations decay as in the usual excitation-deexcitacion cycle, so that excitation disappears at long times. At sufficiently large biological growth rate, however, the steady filament solution exists, is stable, and the initial perturbation can be locally attracted by it. Chaotic flow deforms the 
simple filament solution obtained under simplified assumptions [8, 9], but the results in [8] indicate that it still provides a useful description of the process. Chaotic stretching and folding of the excited filament in a closed system ends up when it fills the whole domain, after which an homogeneous deexcitation finishes the excitation cycle. In an open system, however, the continuous outflow of excited material inhibits the filling of the full domain, so that distributions related to relatively simple filament steady solutions can persist permanently.

\section{Nonlocal logistic growth}

In this section we discuss the interacting particle model with non-local interactions introduced in [10]. It is just a simple modification of the Brownian Bug model of [11] where there is an ensemble of diffusing particles (the bugs), each of them dying or duplicating with given probabilities per unit of time. As we will see below the modification consists in the introduction of an interaction among the particles so that the birth rates for any of them diminish in regions of high particle density. This is precisely Verhulst logistic mechanism, here implemented in a particle model instead that in the original Verhulst equation for the global population.

The microscopic rules are enumerated in the following. Let $N(t)$ be the number of bugs in the system (a two-dimensional periodic box $\Omega$ of size $L \times L$; in all our computer simulations we will take $L=1$ ):

1. There is an initial population of $N(t=0)=N_{0}$ bugs or particles, randomly located.

2. One particle, $j$, is selected at random and it reproduces (i.e., it is transformed into two particles) with a rate (probability per unit time) $\lambda(j)$ or dies with a rate $\beta(j)$. Both rates are not constant but depend on the number of particles surrounding the particle $j$. Explicitly we take:

$$
\lambda(j)=\max \left(0, \lambda_{0}-\frac{1}{N_{s}} N_{R}^{j}\right),
$$

and

$$
\beta(j)=\max \left(0, \beta_{0}-\frac{\alpha}{N_{s}} N_{R}^{j}\right) .
$$

where $\lambda_{0}$ and $\beta_{0}$ are constants. $N_{R}^{j}$ denotes the total number of particles which are at a distance smaller than $R$ from particle $j$ (excluding the particle $j$ itself). $R$ is thus a range of interaction, $N_{s}$ is a saturation parameter, and $\alpha$ controls the asymmetry between its influence on death and on reproduction (in the rest of this paper we take $\alpha=0$ so that only birth rates are modified by the local density of particles). In the case of reproduction, the newborn particle is located at the same place as the 
parent particle. The process is repeated a number of times equal to $N(t)$, so that each particle is checked for birth or reproduction once in average. 3. Each particle moves in random direction a distance drawn from a Gaussian distribution of standard deviation $\sigma$ (this models Brownian motion).

4. When advection is considered, the particles are transported by an external flow to be described later.

5. Time is incremented an amount $\tau=1$, and the algorithm repeats.

Figure 2 shows typical spatial configurations observed at large times under this algorithm. When the maximum growth rate $\mu \equiv \lambda_{0}-\beta_{0}$ is small $\left(\mu<\mu_{c}\right)$, population dies at long times. Above this value of $\mu$, an active phase with a persistent average number of individuals is attained. The nature of the spatial distribution in the active phase depends on the values of the parameters. For large enough value of the effective diffusion coefficient $D \equiv \sigma^{2} / \tau$, the spatial distribution of particles is homogeneous on average, whereas clear clustering occurs for small $D$. As in the Brownian Bug model [11], different clusters are coming from different families (i.e. each cluster is made of descendants of a different initial reproducing individual). But the most striking feature is that they organize in a periodic pattern. The periodicity of the pattern is of the order of $R$, the interaction range. In addition to decreasing $D$, this transition to a periodic organization occurs by increasing $R$ and, for small enough $D$, by increasing $\mu$.

Particle clustering seems to be a rather natural way to make compatible the high local growth at relatively large value of $\mu$, with the reduction of this growth that a too crowded neighborhood would imply: the empty space between the clusters acts as a buffer zone keeping the competition for resources less limiting than in a homogeneous distribution. We believe that this dispersion of the total population in small groups over a large spatial area is a general consequence of the logistic mechanism when applied to particle systems.

We try now to understand this pattern forming process. To this end we write down a mean-field-like description of the model, which completely neglects fluctuations, and check if the clustering instability appears there. The mean-field equation is written in terms of an expected density $\phi(\mathbf{x}, t)$ as follows

$$
\begin{gathered}
\partial_{t} \phi(\mathbf{x}, t)=D \nabla^{2} \phi(\mathbf{x}, t)+ \\
\left(\lambda_{0}-\beta_{0}\right) \phi(\mathbf{x}, t)-\frac{1}{N_{s}} \phi(\mathbf{x}, t) \int_{|\mathbf{x}-\mathbf{r}|<R} d \mathbf{r} \phi(\mathbf{r}, t) .
\end{gathered}
$$

This expression can be understood as a nonlocal version of Verhulst logistic equation, complemented also with the diffusion term arising from the Brownian motion of the bugs. With more generality, the nonlocal term may be written as $\phi(\mathbf{x}, t) \int_{A} d \mathbf{r} G(\mathbf{x}-\mathbf{r}) \phi(\mathbf{r}, t)$, where $A \subset \Omega$. Our model corresponds then to a kernel $G(\mathbf{x})$ given by

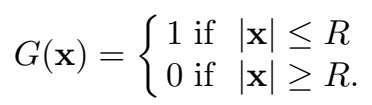



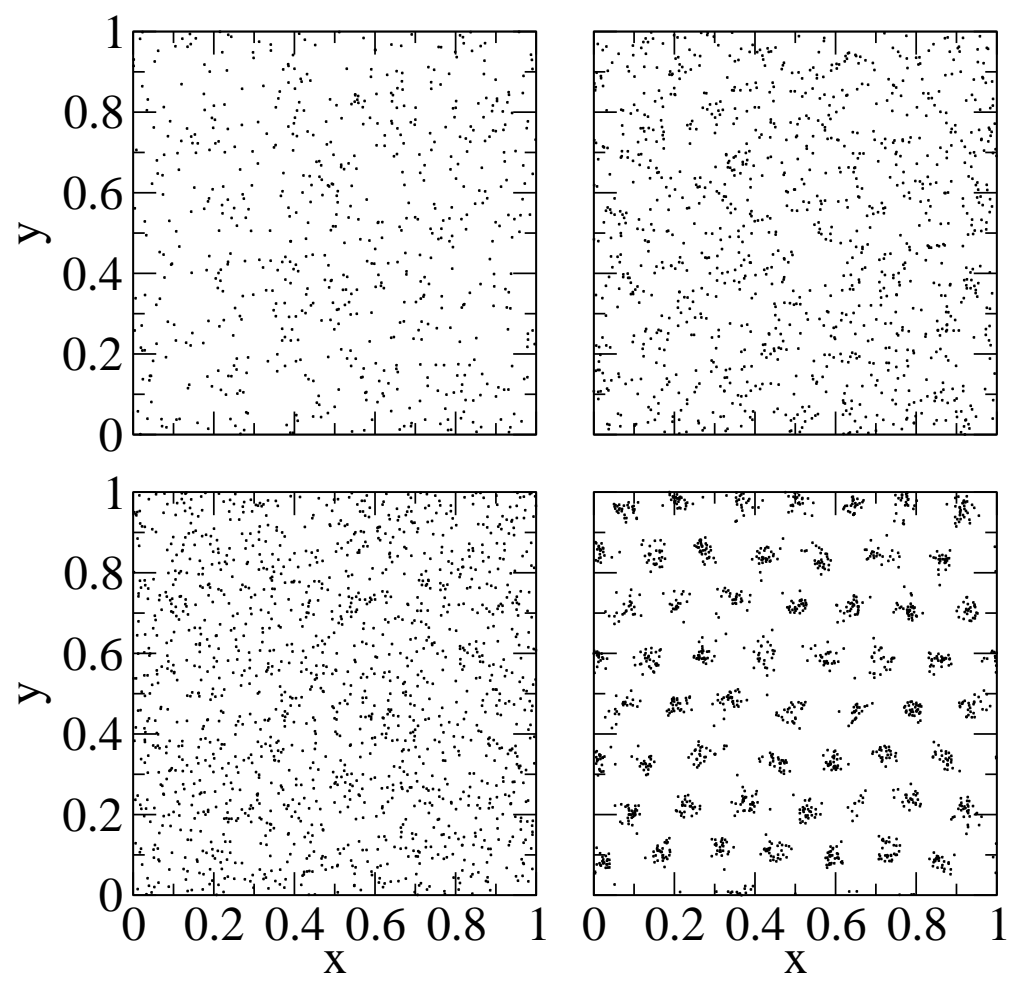

Fig. 2. Long-time spatial structures for the interacting particle model. Left column corresponds to two patterns with the same value of $D=10^{-4}$, and two different values of $\mu=0.5$ (up) and $\mu=0.9$ (bottom). Right column corresponds to fixed $\mu=0.7$, and $D=10^{-4}$ (upper), and $D=10^{-5}$ (bottom). In all the plots, $N_{s}=50$ and $R=0.1$.

Stationary homogenous solutions of equation (7) are the empty phase $\phi(\mathbf{x}, t)=0$, and the active or survival phase $\phi(\mathbf{x}, t)=\phi_{s}=\mu N_{s} / \pi R^{2}$ (remember that $\left.\mu=\lambda_{0}-\beta_{0}\right)$. For $\mu<0$ the only stable solution is the absorbing one; the transition to the survival state is approached at $\mu=0$, and this state is stable for a range of positive values of $\mu$. At the deterministic level the transition is transcritical. We note that the critical value $\mu=0$ is smaller than the one observed numerically in the particle model $\left(\mu_{c}>0\right)$, this deficiency of the mean-field description being a well known consequence of neglecting fluctuations. 
We perform now a stability analysis of the $\phi_{s}$ solution by considering small harmonic perturbations around it, $\phi(\mathbf{x}, t)=\phi_{s}+\delta \phi(\mathbf{x}, t)$, with $\delta \phi(\mathbf{x}, t) \propto$ $\exp (\lambda t+i \mathbf{k} \cdot \mathbf{x})$. After simple calculations (see details in [10]) one arrives to the following dispersion relation

$$
\lambda(K)=-D K^{2}-\frac{2 \mu}{K R} J_{1}(K R),
$$

where $K=|\mathbf{k}|$, and $J_{1}$ is the first-order Bessel function. It is clear that the relevant parameters in the problem are $\mu$ and $D / R^{2}$ (in fact the precise dimensionless combinations are $\mu \tau$ and $D \tau / R^{2}$, see [12]). The eigenvalue $\lambda(K)$ (which is in fact a function of $K R, \mu$, and $D / R^{2}$ ) is real and can be positive for some values of the parameters. This is shown in Fig. (3) where we plot $\lambda$ against $K$ for fixed $D / R^{2}$ and different values of $\mu$ around the critical value $\mu_{P}=185.192 D / R^{2}$, which is the value of $\mu$ at which $\lambda(K)$ becomes positive [10]. Positive values of $\lambda(K)$ in a range of values of $K$ imply instability of the homogeneous distribution against perturbations containing this range of wavenumbers ( $K$ around $K_{c}=4.779 / R$ ) and pattern formation with the corresponding wavelengths $(\approx 1.31 R)$.

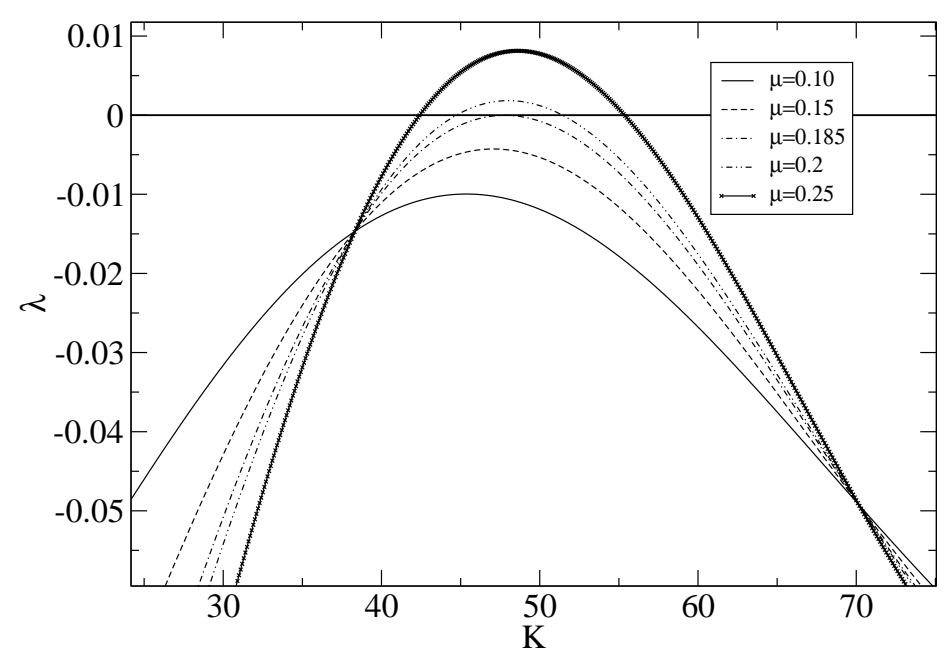

Fig. 3. Linear growth rate $\lambda$ vs wavenumber $K$ from (9) for different values of $\mu$ close to the onset of pattern formation. We take $R=0.1$ and $D=10^{-5}$ so that $\mu_{P}=0.185$.

The behavior of the deterministic Eq. (7) is thus clear: for $\mu<0$ the only stable solution is $\phi=0$. Then there is an interval, $0<\mu<\mu_{P}$, where one has the homogeneous density $\phi=\phi_{s}$, and for $\mu>\mu_{P}$ spatial patterns 
emerge. This last transition can also be crossed by decreasing $D / R^{2}$ at fixed $\mu>0$. The details of this sequence of transitions and the critical parameter values do not coincide with the behavior of the particle model, implying that fluctuations are rather important. However, the presence of a pattern forming instability is well explained and even the selected pattern wavenumber $\left(K_{c} \approx\right.$ $4.779 / R$ ) is quantitatively reproduced by the mean-field approach [10]. Fig. 4 shows a steady pattern of density which is the solution of Eq. (7) reached at long times. It is analogous to the one shown for the discrete model in the bottom-right panel of Fig. 2, confirming for the full nonlinear model (7) the behavior identified from the linear stability analysis of the homogeneous solutions.

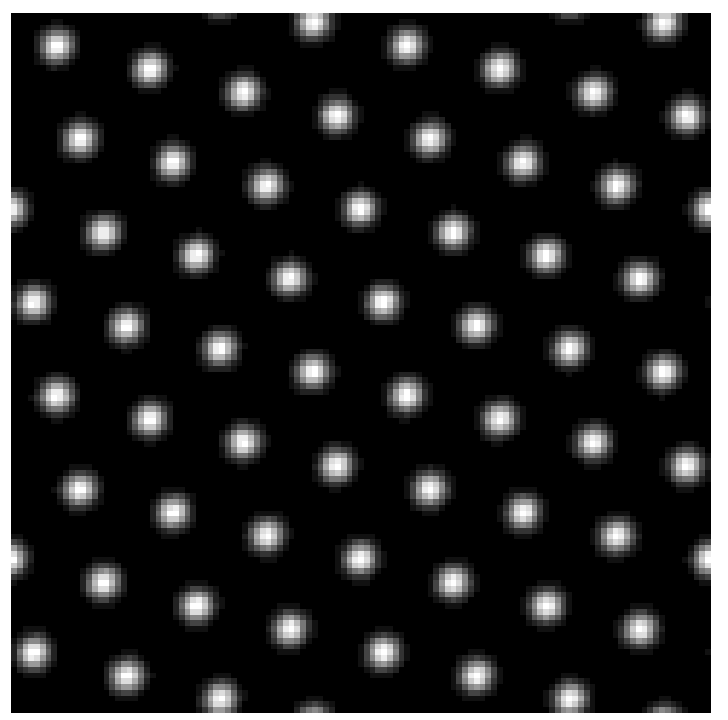

Fig. 4. Steady spatial pattern from the deterministic equation (7). $\mu=0.70$, $R=0.1, D=10^{-5}$, and $N_{s}=50$. Note the strong similarity with the pattern in the bottom-right plot in Fig. 2.

So far the only motion considered for the bugs has been Brownian motion. If they live in a turbulent aquatic medium, they will be also subjected to straining fields that will deform the clusters and, as in the previous Section, alter the population dynamics. We have implemented in the fourth step of the algorithm defining the particle model a simplified flow consisting in shear motions alternating in direction: if we denote by $\left(x_{i}(t), y_{i}(t)\right)$ the coordinates of the particle $i$ at time $t$, after one iteration of the map they become

$$
\begin{aligned}
& x_{i}\left(t^{\prime}\right)=x_{i}(t)+A \cos \left(y_{i}(t)\right), \\
& y_{i}\left(t^{\prime}\right)=y_{i}(t)+A \cos \left(x_{i}\left(t^{\prime}\right)\right) .
\end{aligned}
$$



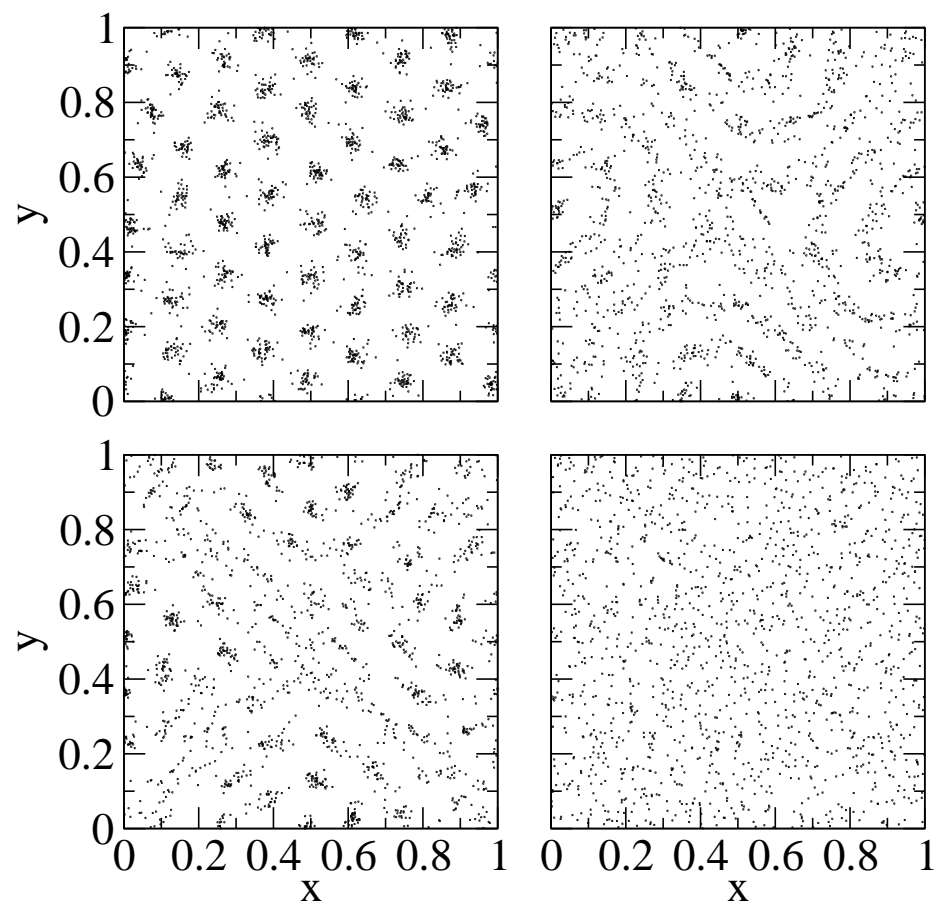

Fig. 5. Snapshots of the long-time spatial structure for the distribution of particles with an external flow. From top to bottom and left to right, $A=0, A=0.1, A=0.5$, and $A=1$. The other parameters: $\mu=0.9, D=5 \times 10^{-6}, N_{s}=50$, and $R=0.1$.

where $t^{\prime}=t+\tau$. The parameter $A$ gives the strength of the flow and, depending of its value, particles can follow regular or chaotic trajectories. Next we analyze the behavior of the model when the flow changes. In Fig. 5 we show instantaneous configurations of the particle distributions as they are being stirred by the flow at different values of $A$. Increasing $A$ leads to increasingly chaotic trajectories and mixing behavior in the flow. It is seen that the periodic array of clusters in the absence of flow becomes more filamentallike as the flow strength increases. The shape of the filamental structures reflects the known unstable and stable foliation of phase space for the map (11). Inhomogeneity persists for rather strong flow, but finally the distribution becomes homogeneized. At this point, the particle distribution should be very close to Poissonian, with density given by the homogeneous solution of (7). This is indeed what is observed in Fig. 6 where we plot time evolution of the total number of particles. For large values of $A$ the total number of particles, $N(t)$, fluctuates around the homogeneous deterministic solution value $\phi_{s}=\mu N_{s} / \pi R^{2}$. For smaller flow strength $A$ the spatial structure in the neighborhood of each particle becomes relevant, and the number of particles approaches the value in the absence of flow, corresponding to the pattern state 
of clustered particles. Thus, we see that the nonlocal interactions lead to a coupling between flow and population dynamics, mediated by the changes in local distribution geometry that the flow induces.

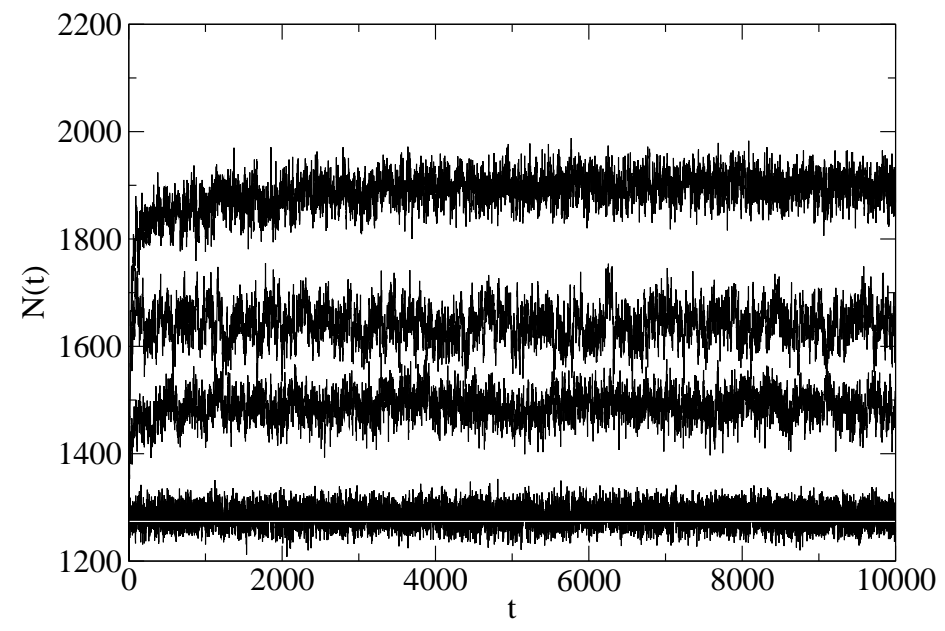

Fig. 6. $N(t)$ vs time for different values of the external flow strength, $A$. From top to bottom: $A=0, A=0.01, A=0.05$, and, fluctuating around the value $\phi_{s}=\mu N_{s} / \pi R^{2}$ (horizontal white line), $A=1$ and $A=3$. The other parameters are $\mu=0.8, N_{s}=50, D=10^{-5}$, and $R=0.1$.

\section{Summary}

In this contribution we have presented results on two model systems coming from the context of population dynamics in aquatic flowing media. In both cases the logistic mechanism of P.F. Verhulst is an essential ingredient, although the presence of nontrivial predation dynamics in one case, and of a finite range of interaction in the other lead to interesting new phenomena, namely excitability and pattern formation, respectively. In both model systems the consideration of fluid flow leading to chaotic trajectories (the process known as chaotic advection or Lagrangian turbulence [13]) has additional impact on the dynamics. 


\section{Acknowledgements}

Financial support from MEC (Spain) and FEDER from grants CONOCE2 (FIS2004-00953) and IMAGEN (REN2001-0802-C02-01/MAR) is greatly acknowledged. C.L. is a Ramón y Cajal fellow.

\section{References}

1. P.F. Verhulst: Corresp. Math. Phys. 10, 113 (1838).

2. K.H. Mann and J.R.N. Lazier: Dynamics of Marine Ecosystems. BiologicalPhysical Interactions in the Oceans (Blackwell Scientific Publications, Boston, 1991).

3. A.P. Martin: Progr. Oceanogr. 57, 125 (2003).

4. E. Hernández-García, C. López, and Z. Neufeld: Spatial Patterns in Chemically and Biologically Reacting Flows. In: Chaos in Geophysical Flows, ed. by G. Bofetta, G. Lacorata, G. Visconti, and A. Vulpiani (OTTO editore, Torino, 2003) pp. 35-61. Available from the publisher website: http://www.otto.to.it/otto/index2_e.php

5. J.E. Truscott and J Brindley, Bull. Math. Biol. 56, 981 (1991).

6. E. Hernández-García and C. López, Ecological Complexity 1, 253 (2004).

7. E. Ott and T. Tél, Chaos 3, 417 (1993).

8. Z. Neufeld, C. López, E. Hernández-García, and O. Piro, Phys. Rev. E 66, 066208 (2002).

9. E. Hernández-García, C. López, and Z. Neufeld, Physica A 327, 59 (2003).

10. E. Hernández-García and C. López, Phys. Rev. E 70, 016216 (2004).

11. W.R. Young, A.J. Roberts, and G. Stuhne, Nature 412, 328 (2001).

12. C. López and E. Hernández-García, Physica D, in press (2004).

13. H. Aref, Phys. Fluids, 14, 1315 (2002). 